Vietnam Journal of Mechanics, VAST, Vol.39, No. 1 (2017), pp. 1 -12

DOI:10.15625/0866-7136/6058

\title{
OPTIMAL DESIGN FOR EIGEN-FREQUENCIES OF A LONGITUDINAL BAR USING PONTRYAGIN'S MAXIMUM PRINCIPLE CONSIDERING THE INFLUENCE OF CONCENTRATED MASS
}

\author{
Bui Hai Le*, Tran Minh Thuy \\ Hanoi University of Science and Technology, Vietnam \\ *E-mail: le.buihai@hust.edu.vn \\ Received September 21, 2015
}

\begin{abstract}
In this paper, the problem of optimal design for eigen-frequencies of a longitudinal bar using Pontryagin's maximum principle (PMP) considering the influence of concentrated mass is presented. The necessary optimality condition when simultaneously maximizing system's eigen frequencies and minimizing system's weight considering the influence of concentrated mass is established by using Maier objective functional in order to control the final state of the objective functional. By considering eigen frequencies as state variables, the analogy coefficient $k$ in the necessary optimality condition is explicitly determined. Numerical results obtained in this paper include: (1) the bar's optimal configurations as well as frequency responses in different cases of objective functions; (2) the Pareto front for the system's first eigen frequency and weight; (3) the influence of concentrated mass on the bar's optimal configuration.
\end{abstract}

Keywords: Eigen frequencies, optimal design, longitudinal bar, concentrated mass, Pareto front, Pontryagin's maximum principle.

\section{INTRODUCTION}

Bars in longitudinal vibration may be adequately described by one-dimensional continuous models [1]. Longitudinal vibration of bars could be easily found in ultrasonic transducers, motors and machines or in measuring systems to determine material properties [2-5]. Therefore, determination of optimal eigen-frequencies (or eigen-values) as well as configurations will provide useful solutions in practical applications of bars in longitudinal vibration.

Pontryagin's Maximum Principle (PMP) has been widely used to investigate the optimal design for eigen-values of mechanical systems such as for thin walled I beams $[6,7]$ or columns/rods [8-18] with different types of boundary and loading conditions. However, several limitations are still found from the literature as below [19-21]:

(C) 2017 Vietnam Academy of Science and Technology 
i) The optimal shape of a column of greatest efficiency was not determined directly by maximizing column's eigenvalues. Main reason is the fact that column's eigenvalues were not considered as state variables; hence they were not included in objective functions. Consequently, the optimal problems could only be solved by giving column's eigenvalues and minimizing column's volume.

ii) Column's cross-sectional shapes, which were considered as control/design variables, were subjected to unbounded and continuous variation.

iii) Optimal problems were performed with a single objective by minimizing the column's volume.

In our recent work [19-21], the problems of multi-objective optimal design for eigen-frequencies of torsional shafts using Pontryagin's maximum principle were presented with main contributions as follows:

i) The proposition of multi-objective optimal design for eigen-frequencies and weight of circular bars under free torsional and longitudinal vibration using Pontryagin's maximum principle is written in general and dimensionless form and it is proved for all cases of boundary conditions of the bars. The analogy coefficient $k$ in the necessary optimality condition is explicitly determined by considering eigen frequencies as state variables, namely the state equations include eigen frequencies.

ii) Numerical simulations demonstrate the relationship between the optimal configuration of the bar and their eigen modes. It is shown that optimal design of the bar can be roughly carried out with the help of this relationship.

iii) The Pareto fronts and the boundary of the feasible region of objectives, eigenfrequencies and weight of the bar, are constructed. These facilitate the estimation of the level of the trade-off between objectives, and the selection of a suitable solution among a set of competitive objectives.

iv) Equivalents about optimal shapes and eigen frequencies under specific boundary conditions is investigated for bars of different lengths.

v) Bar's cross-sectional diameters, which were considered as control/design variables, can be subjected to bounded and discontinuous variation.

In the present work, PMP is used to optimal design for eigen-frequencies of a circular bar in free longitudinal vibration considering the influence of concentrated mass.

\section{PROBLEM UNDER CONSIDERATION}

A circular bar of length $L$ containing $n$ concentrated masses $m_{e}(e=1 \div n)$ is subdivided into $n-1$ segments (elements) as depicted in Fig. $1 . L_{e}$ and $d_{e}$ are length and diameter of $i^{\text {th }}$ element, respectively. The finite element model (FEM) of the bar includes $n$ nodes $(1,2, \ldots, e, \ldots, n-1, n)$ and $(n-1)$ elements $(\mathbf{1}, 2, \ldots, \boldsymbol{e}, \ldots, \boldsymbol{n}-\mathbf{2}, \boldsymbol{n}-\mathbf{1})$.

The governing equation of a bar in free longitudinal vibration is written under the following form

$$
E A \frac{d^{2} u(x)}{d x^{2}}+\rho A \omega^{2} u(x)=0,
$$

where, $E$ and $\rho$ are the bar material's elastic modulus and mass density, respectively. 


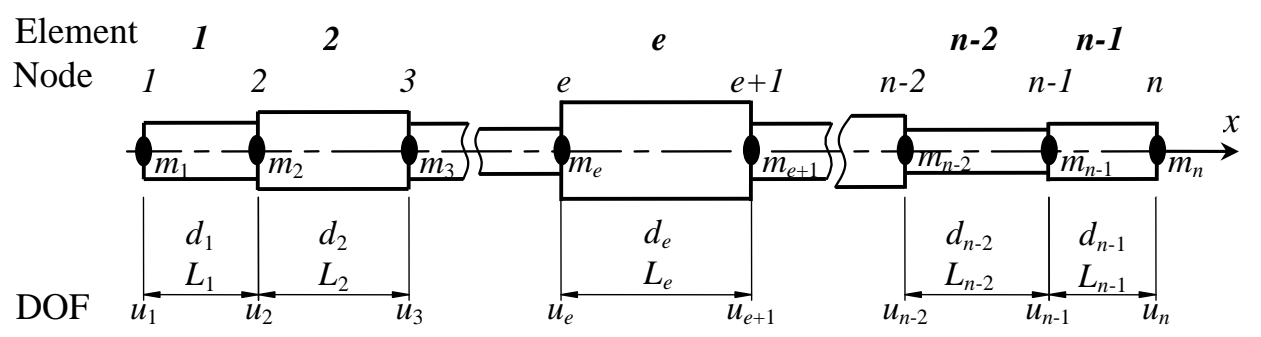

Fig. 1. The model of the circular bar containing concentrated masses

$u(x)$ is the displacement at position $x . A$ is the cross-sectional area. $\omega$ is the bar's eigen frequency. Eq. (1) can be rewritten in the form of state differential equations as ( $N$ is the internal force amplitude)

$$
\left\{\begin{array}{l}
\frac{d u}{d x}=\frac{N}{E A} \\
\frac{d N}{d x}=-\rho A \omega^{2} u
\end{array}\right.
$$

At the section containing the concentrated mass $m$, the equilibrium and continuum conditions are expressed as

$$
\left\{\begin{array}{l}
N_{+}=N_{-}-m \omega^{2} u \\
u_{+}=u_{-}
\end{array}\right.
$$

where $N_{+}$and $u_{+}, N_{-}$and $u_{-}$are the internal forces and the displacements on the right and left of the considered section, respectively. The fixed - free boundary condition is considered as follows

$$
u(0)=0, \quad N(L)=0 .
$$

Eqs. (2) can be easily solved by finite element method (FEM) with matrix of dynamic rigidity using Matlab software.

\section{MULTI-OBJECTIVE OPTIMAL DESIGN OF THE CIRCULAR BAR IN FREE LONGITUDINAL VIBRATION}

PMP is here used to multi-objective optimal design of the circular bar in free longitudinal vibration, in which maximizing system's eigen frequencies and minimizing system's weight are simultaneously involved Optimization problem is stated as follows: find $d_{e}(e=1 \div n-1), d_{e} \in\left[d_{\min }, d_{\max }\right]$, which satisfies the objective function $F$

$$
F=-\left(1-k_{\omega W}\right) \frac{\omega_{i}}{\omega_{0 i}}+k_{\omega W} \frac{W}{W_{0}} \rightarrow \min
$$

where $\omega_{i}$ is the $i^{t h}$ natural frequency, $k_{\omega W}$ is non-negative weight, $k_{\omega W} \in[0,1), W$ is the total weight of the bar, $\omega_{0 i}$ and $W_{0}$ are the $i^{\text {th }}$ natural frequency and the total weight of the initial bar (before optimizing), respectively.

Based on the state differential equations (2); the objective function $F$, Eq. (5) as well as the boundary condition, Eq. (4); the following proposition is needed for solving the multi-objective optimal design problem. 
Proposition: with the above-mentioned suppositions, Eqs. (2), (4) and (5), the Hamiltonian function $H$ is maximized, and the analogy coefficient $k$ between adjoint variables and original variables is positive, where

$$
H=\frac{1}{k}\left(-\frac{N^{2}}{E A}-\rho A \omega_{i}^{2} u^{2}\right)-\frac{k_{\omega W}}{W_{0}} \rho A \rightarrow \max (\text { in } \mathrm{A})
$$

Proof:

The natural frequency $\omega_{i}$ is here considered as a state variable. It means that the role of $\omega_{i}$ is equivalent to those of $u$ and $N$ in the state Eqs. (2). The total weight $W$ is also a state variable. Hence, the state equations (2) can be rewritten in the form

$$
\begin{aligned}
& \frac{d u}{d x}=\frac{N}{E A}, \\
& \frac{d N}{d x}=-\rho A \omega_{i}^{2} u, \\
& \frac{d \omega_{i}}{d x}=0, \\
& \frac{d W}{d x}=\rho A .
\end{aligned}
$$

The equilibrium and continuum conditions at the section containing the concentrated masses $m$ are given as below

$$
\left\{\begin{array}{l}
N_{+}=N_{-}-m \omega_{i}^{2} u \\
u_{+}=u_{-}=u
\end{array}\right.
$$

Thus,

$$
\left\{\begin{array}{l}
\delta N_{+}=\delta N_{-}-m \omega_{i}^{2} \delta u \\
\delta u_{+}=\delta u_{-}=\delta u
\end{array}\right.
$$

The objective function (5) can be rewritten in term of the Maier objective functional as follows

$$
F=-\left(1-k_{\omega W}\right) \frac{\omega_{i}(L)}{\omega_{0 i}}+k_{\omega W} \frac{W(L)}{W_{0}} \rightarrow \min
$$

The Hamiltonian function $H$ can be established in the form as follows

$$
H=p_{u} \frac{N}{E A}-p_{N} \rho A \omega_{i}^{2} u+p_{\omega} \omega_{i}^{\prime}+p_{W} \rho A, \quad\left(\omega_{i}^{\prime}=0\right)
$$

The adjoint equations can be expressed in the following form

$$
\begin{gathered}
\frac{d p_{u}}{d x}=-\frac{\partial H}{\partial u}=\rho A \omega_{i}^{2} p_{N}, \\
\frac{d p_{N}}{d x}=-\frac{\partial H}{\partial N}=-\frac{1}{E A} p_{u}, \\
\frac{d p_{\omega}}{d x}=-\frac{\partial H}{\partial \omega_{i}}=2 \rho_{i} A_{i} \omega_{i} u p_{N},
\end{gathered}
$$




$$
\frac{d p_{W}}{d x}=-\frac{\partial H}{\partial W}=0 .
$$

The adjoint variables $p_{u}, p_{N}, p_{\omega}, p_{W}$ are determined from the expression

$$
\sum_{i=1}^{n} p_{i}(L) \delta x_{i}(L)-\sum_{i=1}^{n} p_{i}(0) \delta x_{i}(0)+\delta F(L)=0,
$$

where $x_{i}, p_{i}$ are state and adjoint variables, respectively.

Thus,

$$
\begin{gathered}
p_{u}(L) \delta u(L)+p_{N}(L) \delta N(L)+p_{\omega}(L) \delta \omega_{i}(L)+p_{W}(L) \delta W(L)-p_{u}(0) \delta u(0) \\
-p_{N}(0) \delta N(0)-p_{\omega}(0) \delta \omega_{i}(0)-p_{W}(0) \delta W(0)+p_{N+} \delta N_{+}+p_{u+} \delta u_{+} \\
-p_{N-} \delta N_{-}-p_{u-} \delta u_{-}-\left(1-k_{\omega W}\right) \frac{\delta \omega_{i}(L)}{\omega_{0 i}}+k_{\omega W} \frac{\delta W(L)}{W_{0}}=0 .
\end{gathered}
$$

Or,

$$
\begin{aligned}
p_{u}(L) & \delta u(L)+p_{N}(L) \delta N(L)+\left[p_{\omega}(L)-\frac{1-k_{\omega W}}{\omega_{0 i}}\right] \delta \omega_{i}(L)+\left[p_{W}(L)+\frac{k_{\omega W}}{W_{0}}\right] \delta W(L) \\
& -p_{u}(0) \delta u(0)-p_{N}(0) \delta N(0)-p_{\omega}(0) \delta \omega_{i}(0)-p_{W}(0) \delta W(0) \\
& +\delta N_{-}\left(p_{N+}-p_{N-}\right)+\left[p_{u+}-p_{u-}-p_{N+} m \omega_{i}^{2}\right] \delta u=0 .
\end{aligned}
$$

We obtain

$$
\begin{aligned}
& \left\{\begin{array}{l}
p_{u}(L)=0 \\
p_{\omega}(L)=\frac{1-k_{\omega W}}{\omega_{0 i}} \\
p_{W}(L)=-\frac{k_{\omega W}}{W_{0}} \\
p_{N}(0)=0 \\
p_{\omega}(0)=0 \\
p_{W}(0)=0
\end{array}\right. \\
& p_{u+}=p_{u-}+p_{N+} m \omega_{i}^{2}, \\
& p_{N+}=p_{N-} .
\end{aligned}
$$

Assigning

$$
\left\{\begin{array}{l}
p_{u}=-N_{H} \\
p_{N}=u_{H}
\end{array}\right.
$$

Combining Eqs. (11a), (11b) and (16) yields

$$
\begin{aligned}
\frac{d u_{H}}{d x} & =\frac{N_{H}}{E A}, \\
\frac{d N_{H}}{d x} & =-\rho A \omega_{i}^{2} u_{H} .
\end{aligned}
$$

Eqs. (15) can be rewritten as follows

$$
\begin{aligned}
& u_{H}(0)=0, \\
& N_{H}(L)=0 .
\end{aligned}
$$




$$
\begin{aligned}
& N_{H+}=N_{H-}-m \omega_{i}^{2} u_{H}, \\
& u_{H+}=u_{H-} .
\end{aligned}
$$

It is seen that Eqs. (7a) and (7b) are similar in form as those of (17) and the boundary conditions (4) and the equilibrium and continuum conditions at the considered section (8a) are also similar in form as the conditions (18a) and (18b), respectively.

As a result, we reached the following conclusion: the same analogy between the adjoint variables and the original variables holds, or

$$
\begin{aligned}
& k N_{H}=N, \\
& k u_{H}=u .
\end{aligned}
$$

Explicit expression of $k$ can be determined by integrating the Eq. (11c) with appropriate conditions in Eqs. (15)

$$
\int_{0}^{L} p_{\omega}^{\prime} d x=p_{\omega}(L)-p_{\omega}(0)=\frac{1-k_{\omega W}}{\omega_{0 i}}=\frac{2 \rho \omega_{i}}{k} \int_{0}^{L} A u^{2} d x .
$$

Hence,

$$
k=\frac{2 \rho \omega_{i} \omega_{0 i}}{1-k_{\omega W}} \int_{0}^{L} A u^{2} d x>0 .
$$

Thus, the sign of the analogy coefficient $k$ is positive for the case of maximizing $\omega_{i}$. It was demonstrated by considering the natural frequency $\omega_{i}$ as a state variable. The Hamiltonian function (10) will be maximized if the condition (6) corrects.

It is noted that $k_{\omega W} \in[0,1)$, in the case of $k_{\omega W}=1$, the objective function (5) becomes $W=\min$, a trivial solution could be found with $d_{e}=d_{\min }$. If the objective of weight is not needed, $k_{\omega W}=0$.

Thus, basing on the PMP in optimal design for above-mentioned system's natural frequency and total weight containing the influence of concentrated masses, the obtained optimal necessary conditions consist of: the state Eqs. (2), the boundary conditions (4), the design vector $d_{e} \in\left[d_{\min }, d_{\max }\right]$ and the maximum condition of the Hamiltonian function (6).

\section{RESULTS AND DISCUSSIONS}

A fixed-free circular bar in free longitudinal vibration is considered with the elastic modulus and $E=200 \mathrm{GPa}$ and mass density $\rho=7845 \mathrm{~kg} / \mathrm{m}^{3}$. The bar is divided by $n-1$ elements ( $n$ nodes). Element length is $L_{e}=L /(n-1)$, where $L=2 \mathrm{~m}$ is the bar's length. The initial bar diameter is taken as $d_{0}=L / 10$. During optimization, the bar's shape might change and element diameter $d_{e}$ varies in the range $d_{e} / d_{0} \in[0.5,1]$.

\subsection{Optimal design for eigen frequencies of the bar}

Studied cases with the different objective functions and necessary optimality conditions (NOC) are presented in Tab. 1.

Four dimensionless ratios are here defined as below: 
Table 1. Studied cases

\begin{tabular}{|c|c|c|c|}
\hline Case & $d_{e}(e=1 \div 20), \mathrm{m}$ & Objective function & NOC (in $A)$ \\
\hline Case 1 & 0.2 & & \\
\hline Case 2 & {$[0.1,0.2]$} & $\omega_{1} \rightarrow \max$ & $-\frac{N^{2}}{E A}-\rho A \omega_{1}^{2} u^{2} \rightarrow \max$ \\
\hline Case 3 & {$[0.1,0.2]$} & $\omega_{2} \rightarrow \max$ & $-\frac{N^{2}}{E A}-\rho A \omega_{2}^{2} u^{2} \rightarrow \max$ \\
\hline Case 4 & {$[0.1,0.2]$} & $\omega_{1} \rightarrow \min$ & $\frac{N^{2}}{E A}+\rho A \omega_{1}^{2} u^{2} \rightarrow \max$ \\
\hline Case 5 & {$[0.1,0.2]$} & $\omega_{2} \rightarrow \min$ & $\frac{N^{2}}{E A}+\rho A \omega_{2}^{2} u^{2} \rightarrow \max$ \\
\hline
\end{tabular}

$$
R_{\omega 1}=\frac{\omega_{1}-\omega_{01}}{\omega_{01}}, R_{\omega 2}=\frac{\omega_{2}-\omega_{02}}{\omega_{02}}, R_{\omega 12}=\frac{\left(\omega_{2}-\omega_{1}\right)-\left(\omega_{02}-\omega_{01}\right)}{\omega_{02}-\omega_{01}}, R_{w}=\frac{W-W_{0}}{W_{0}}
$$

where, $\omega_{01}, \omega_{02}$ and $W_{0}$; and $\omega_{1}, \omega_{2}$ and $W$ are the first and second frequencies and total weight of the bar in cases $d_{e}=d_{0}$ and optimal cases, respectively.

Variations of the bar's optimal configurations versus the bar's dimensionless length, $l / L$ with $l \in[0, L]$, are presented in Fig. 2 . It can be seen from Fig. 2 that in the cases of maximizing bar's eigen frequencies the bar's optimal configurations are gradually changed, whereas in the cases of minimizing bar's eigen frequencies the variations of the bar's optimal configurations are in pulse shapes.

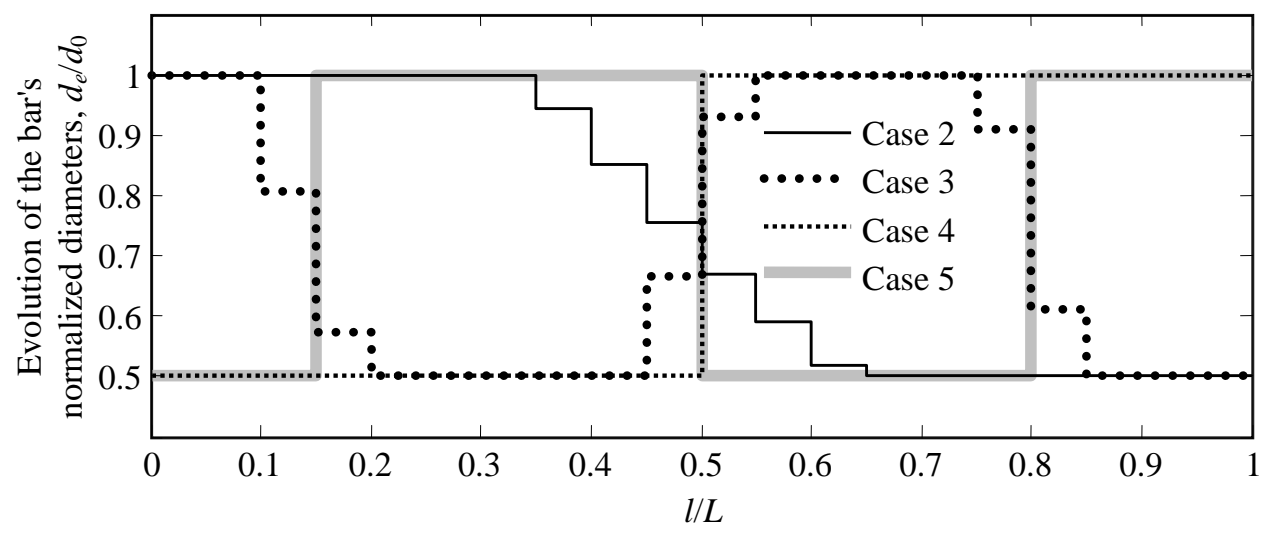

Fig. 2. The bar's optimal configurations in studied cases

Variations of $R_{\omega 1}, R_{\omega 2}, R_{\omega 12}, R_{W}$ in cases $2,3,4,5$ are plotted in Fig. 3. Compared to case $1, \omega_{1}$ and $\omega_{2}$ vary from $-41 \%$ to $44 \%$, and from $-41 \%$ to $42 \%$, respectively. Reduction in the weight of the optimal bar falls in the range $34 \% \div 43 \%$ in cases $2-5$. For example, in case $2\left(\omega_{1} \rightarrow \max \right), \omega_{1}$ increases $44 \%$ and $W$ decreases $22 \%$. The difference 
between $\omega_{2}$ and $\omega_{1},\left(\omega_{2}-\omega_{1}\right)$, reduces $34 \%$ from that in case 1 . Difference between the first and second frequency is maximal in case 3 , the ratio $R_{\omega 12}$ reaches a maximal value, about $71 \%$ and $\omega_{2}-\omega_{1}=13634 \mathrm{rad} / \mathrm{s}$. $\omega_{1}$ increases and $\omega_{2}$ decreases in case 2 , whereas the inverse phenomenon is observed in cases 3 and 4 .

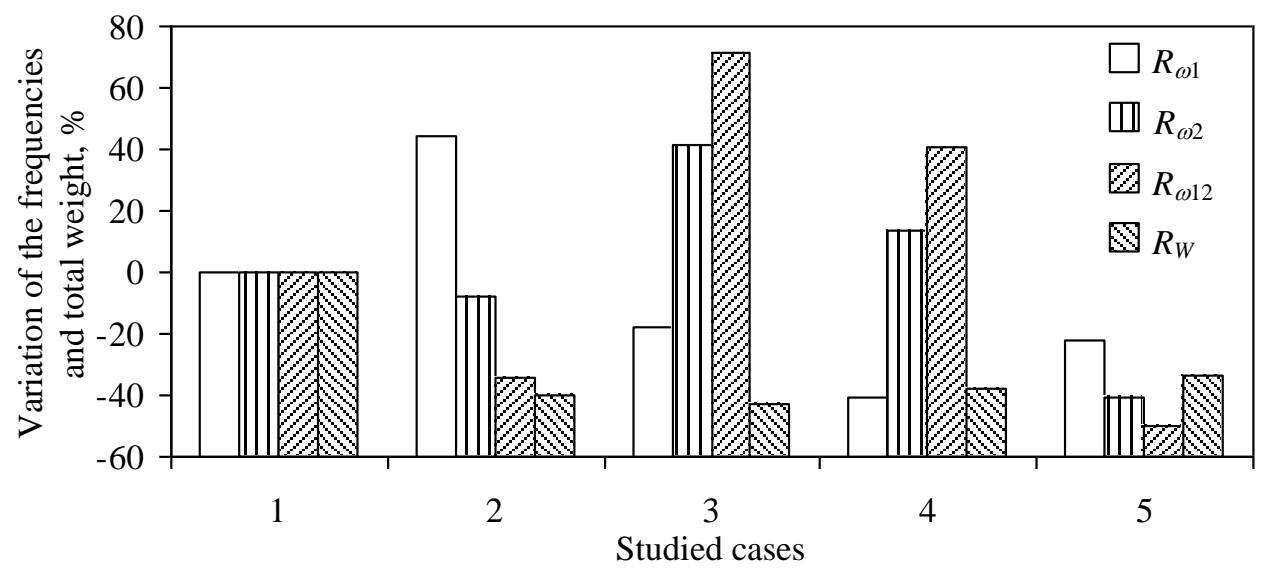

Fig. 3. Relative variation of $\omega_{1}, \omega_{2}, \omega_{2}-\omega_{1}$ and $W$ in studied cases

Frequency responses of the bar subjected to a harmonic axial force at the free end of the bar in the studied cases are presented in Fig. 4, where $f$ is the frequency of the force. It is emphasized from Fig. 4 that appropriate configuration of the bar can be found based on its working region and different purposes in using.

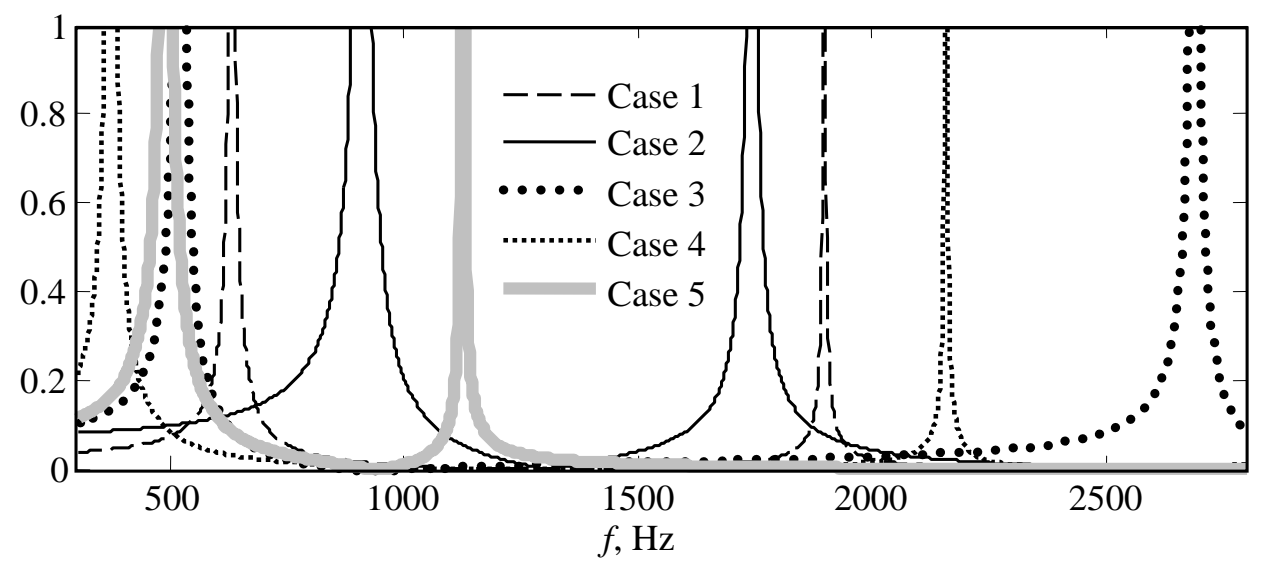

Fig. 4. Frequency responses in studied cases

\subsection{Multi-objective optimal design for the first eigen frequency and total weight of the bar}

In this subsection, the problem of multi-objective optimal design for the first eigen frequency and total weight of the bar is considered. Hence, the necessary optimality 
condition, Eq. 6, becomes

$$
H=\frac{1}{k}\left(-\frac{N^{2}}{E A}-\rho A \omega_{1}^{2} u^{2}\right)-\frac{k_{\omega W}}{W_{0}} \rho A \rightarrow \max , \quad(\text { in } \mathrm{A})
$$

where $k=\frac{2 \rho \omega_{1} \omega_{01}}{1-k_{\omega W}} \int_{0}^{L} A u^{2} d x, \omega_{01}$ and $W_{0}$ are respectively the first eigen frequency and total weight of the bar in Case 1 (subsection 4.1).

From the multi-criteria optimization viewpoint, the Pareto front between the criteria $\left(-\omega_{1} \rightarrow \min\right.$ and $\left.W \rightarrow \min \right)$ is constructed according to the definition 6 in the book by Coello et al. [22]. This definition says that $x^{*}$ is Pareto-optimal if there exists no feasible vector $x$ which would decrease some criterion without causing a simultaneous increase in at least one other criterion (assuming minimization). Pareto front (or trade-off curve), which include the set of points that bounds the boundary of the feasible region, of two objectives is shown in Fig. 5.

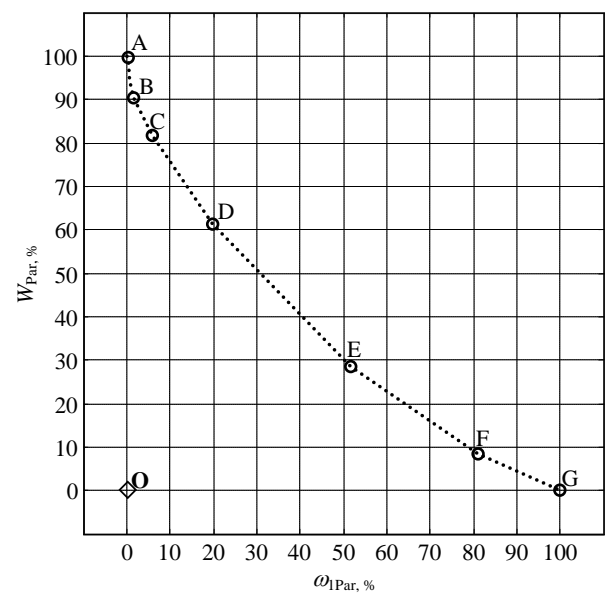

Fig. 5. Pareto front of two objectives $-\omega_{1} \rightarrow \min$ and $W \rightarrow \min$

In which, $\omega_{1 \text { Par }}(\%)$ and $W_{\text {Par }}(\%)$ are the normalized variation of the maximum value of the first eigen frequency and the total weight of the bar, respectively. $\omega_{1 \text { up }}$, $\omega_{1 l o w}, W_{u p}$ and $W_{\text {low }}$ are the biggest and smallest value of the first eigen frequency and the total weight of the bar, respectively. $\omega_{1 E x}$ and $W_{E x}$ are respectively the extremum values of the first eigen frequency and the total weight of the bar determined from the optimal condition, Eq. (23).

$$
\omega_{1 \text { Par }}=\frac{100\left(\omega_{1 \mathrm{up}}-\omega_{1 \mathrm{Ex}}\right)}{\omega_{1 \mathrm{up}}-\omega_{1 \mathrm{low}}}, \quad W_{\text {Par }}=\frac{100\left(W_{\mathrm{Ex}}-W_{\text {low }}\right)}{W_{\mathrm{up}}-W_{\text {low }}} .
$$

From Fig. 5 it is seen clearly that all objectives can never be simultaneously reached in best possibility or expected solution (point 0). The Pareto front allows evaluating dominated and non-dominated Pareto optimal solutions and it also represents the possible 
trade-off levels among different objectives $\left(-\omega_{1} \rightarrow \min\right.$ and $\left.W \rightarrow \min \right)$ as explained in Tab. 2. These results could be used as reference data for designers when designing bars in free longitudinal vibration subjected to simultaneous constraints of eigen frequencies and total weight of the system.

Table 2. Trade-off levels among different objectives

\begin{tabular}{|c|c|c|c|}
\hline Segment & Variation of $\omega_{1 \text { Par }}, \%$ & Variation of $W_{\text {Par }}, \%$ & Higher performance in optimizing \\
\hline $\mathrm{AB}$ & 1.6 & -9.7 & $W$ \\
\hline $\mathrm{BC}$ & 4.3 & -8.5 & $W$ \\
\hline $\mathrm{CD}$ & 13.9 & -20.3 & $W$ \\
\hline $\mathrm{DE}$ & 21.8 & -23 & $W$ \\
\hline $\mathrm{EF}$ & 29.4 & -20.9 & $\omega_{1}$ \\
\hline FG & 18.8 & -8.3 & $\omega_{1}$ \\
\hline
\end{tabular}

\subsection{The influence of the concentrated mass on the bar's optimal configurations}

In order to study the influence of the concentrated mass on the bar's optimal configurations, the bar containing a concentrated mass at the node $11\left(m_{11}\right)$ is considered. The bar's optimal configurations in the case of $\omega_{1} \rightarrow$ max with different values of $m_{11}$ are plotted in Fig. 6.

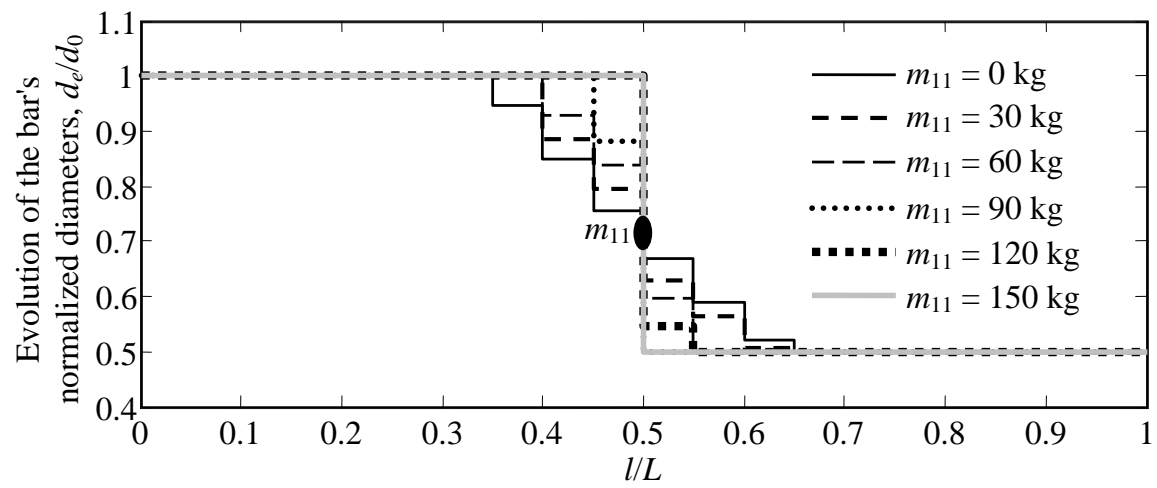

Fig. 6. The influence of the concentrated mass on the bar's optimal configurations in the case of $\omega_{1} \rightarrow \max$

It can be found from Fig. 6 that, the concentrated mass $m_{11}$ has significant influence on the bar's optimal configuration. When increasing the value of $m_{11}$, the different among diameters of the bar's segments also increases. When $m_{11}$ is equal to or higher than a critical value (about $150 \mathrm{~kg}$ ), the bar's optimal configuration is in the pulse shape with only two steps and the transitional point coincides with the location of the concentrated mass $m_{11}$. 


\section{CONCLUSIONS}

In this paper, the problem of optimal design for eigen-frequencies of a longitudinal bar using Pontryagin's maximum principle (PMP) considering the influence of concentrated mass is presented. The main results are summarized as follows:

- The proposition of multi-objective optimal design for eigen-frequencies and total weight of a longitudinal bar using PMP considering the influence of concentrated mass is written and demonstrated in dimensionless form. The analogy coefficient $k$ in the necessary optimality condition is explicitly determined by considering eigen frequencies as state variables, namely the state equations include eigen frequencies.

- Numerical simulations present the variations of for eigen-frequencies and total weight of the bar in the cases of maximizing and minimizing system's eigen frequencies as well as the bar's frequency responses in different cases of objective functions.

- The Pareto front of the objectives $\left(-\omega_{1} \rightarrow \min\right.$ and $\left.W \rightarrow \min \right)$ is constructed. It facilitate the estimation of the level of the trade-off between objectives, and the selection of a suitable solution among a set of competitive objectives. studied.

- The influence of the concentrated mass on the bar's optimal configuration is also

Results can be easily extended to other boundary conditions, higher frequencies of the bar as well as to differential structures.

\section{ACKNOWLEDGEMENTS}

This research is funded by Vietnam National Foundation for Science and Technology Development (NAFOSTED) under grant number 107.01-2015.10.

\section{REFERENCES}

[1] A. DasGupta and P. Hagedorn. Vibrations and waves in continuous mechanical systems. Wiley, New York, (2007). doi:10.1002/9780470518434.

[2] Y. Yi, W. Seemann, R. Gausmann, and J. Zhong. Development and analysis of a longitudinal and torsional type ultrasonic motor with two stators. Ultrasonics, 43, (8), (2005), pp. 629-634. doi:10.1016/j.ultras.2005.03.007.

[3] V. K. Astashev and V. I. Babitsky. Ultrasonic processes and machines: dynamics, control and applications. Springer Science \& Business Media, (2007). doi:10.1007/978-3-540-72061-4.

[4] H. Al-Budairi, M. Lucas, and P. Harkness. A design approach for longitudinaltorsional ultrasonic transducers. Sensors and Actuators A: Physical, 198, (2013), pp. 99-106. doi:10.1016/j.sna.2013.04.024.

[5] Y. Kubojima and S. Sonoda. Measuring Youngs modulus of a wooden bar using longitudinal vibration without measuring its weight. European Journal of Wood and Wood Products, 73, (3), (2015), pp. 399-401. doi:10.1007/s00107-015-0884-2.

[6] C. Szymczak. Optimal design of thin walled I beams for extreme natural frequency of torsional vibrations. Journal of Sound and Vibration, 86, (2), (1983), pp. 235-241. doi:10.1016/0022460x(83)90751-4.

[7] C. Szymczak. Optimal design of thin-walled I beams for a given natural frequency of torsional vibrations. Journal of Sound and Vibration, 97, (1), (1984), pp. 137-144. doi:10.1016/0022460x(84)90474-7. 
[8] T. M. Atanackovic and S. S. Simic. On the optimal shape of a Pflüger column. European Journal of Mechanics-A/Solids, 18, (5), (1999), pp. 903-913. doi:10.1016/s0997-7538(99)00128-x.

[9] V. B. Glavardanov and T. M. Atanackovic. Optimal shape of a twisted and compressed rod. European Journal of Mechanics-A/Solids, 20, (5), (2001), pp. 795-809. doi:10.1016/s09977538(01)01165-2.

[10] T. M. Atanackovic and D. J. Braun. The strongest rotating rod. International Journal of NonLinear Mechanics, 40, (5), (2005), pp. 747-754. doi:10.1016/j.ijnonlinmec.2004.09.002.

[11] T. M. Atanackovic and B. N. Novakovic. Optimal shape of an elastic column on elastic foundation. European Journal of Mechanics-A/Solids, 25, (1), (2006), pp. 154-165. doi:10.1016/j.euromechsol.2005.06.008.

[12] T. M. Atanackovic. Optimal shape of a strongest inverted column. Journal of Computational and Applied Mathematics, 203, (1), (2007), pp. 209-218. doi:10.1016/j.cam.2006.03.019.

[13] T. M. Atanackovic. Optimal shape of a rotating rod with unsymmetrical boundary conditions. Journal of Applied Mechanics, 74, (6), (2007), pp. 1234-1238. doi:10.1115/1.2744041.

[14] Z. D. Jelicic and T. M. Atanackovic. Optimal shape of a vertical rotating column. International Journal of Non-Linear Mechanics, 42, (1), (2007), pp. 172-179. doi:10.1016/j.ijnonlinmec.2006.10.020.

[15] D. J. Braun. On the optimal shape of compressed rotating rod with shear and extensibility. International Journal of Non-Linear Mechanics, 43, (2), (2008), pp. 131-139. doi:10.1016/j.ijnonlinmec.2007.11.001.

[16] T. M. Atanackovic, B. B. Jakovljevic, and M. R. Petkovic. On the optimal shape of a column with partial elastic foundation. European Journal of Mechanics-A/Solids, 29, (2), (2010), pp. 283289. doi:10.1016/j.euromechsol.2009.08.003.

[17] V. B. Glavardanov, D. T. Spasic, and T. M. Atanackovic. Stability and optimal shape of Pflüger micro/nano beam. International Journal of Solids and Structures, 49, (18), (2012), pp. 2559-2567. doi:10.1016/j.ijsolstr.2012.05.016.

[18] T. M. Atanackovic, B. N. Novakovic, and Z. Vrcelj. Shape optimization against buckling of micro-and nano-rods. Archive of Applied Mechanics, 82, (10-11), (2012), pp. 1303-1311. doi:10.1007/s00419-012-0661-1.

[19] M.-Q. Le, D.-T. Tran, and H.-L. Bui. Optimal design of a torsional shaft system using Pontryagins maximum principle. Meccanica, 47, (5), (2012), pp. 1197-1207. doi:10.1007/s11012011-9504-3.

[20] H.-L. Bui, D.-T. Tran, M.-Q. Le, and M.-T. Tran. Multi-objective optimal control for eigenfrequencies of a torsional shaft using Pontryagins maximum principle. Meccanica, 50, (9), (2015), pp. 2409-2419. doi:10.1007/s11012-015-0162-8.

[21] H.-L. Bui, M.-T. Tran, M.-Q. Le, and D.-T. Tran. Optimal configurations of circular bars under free torsional and longitudinal vibration based on Pontryagins maximum principle. Meccanica, 51, (6), (2016), pp. 1491-1502. doi:10.1007/s11012-015-0324-8.

[22] C. C. Coello, G. B. Lamont, and D. A. Van Veldhuizen. Evolutionary algorithms for solving multi-objective problems. Springer Science \& Business Media, (2007). doi:10.1007/978-0-38736797-2. 